\title{
TETANUS - A REVIEW OF CURRENT CONCEPTS IN MANAGEMENT
}

\author{
*O. A. Ogunrin
}

*Neurology Unit, Department of Medicine, University of Benin Teaching Hospital

Benin City, Nigeria

Correspondence:

Dr A. O. Ogunin

Neurology unit, Department of Medicine

University of Benin

PMB 1154 Benin City, Edo State

Nigeria

E-mail: bfunmi@uniben.edu

\section{ABSTRACT}

Tetanus is a vaccinepreventable disease that yearly causes a total of 309,000 deaths. Reports showed up to 1 million cases annually, mostly in underdeveloped countries. Clostridium tetani, the causative organism, is widespread in the faeces of domestic animals and humans, while spores of $C$. tetani are abundant in soil and in the environment surrounding the habitation of humans and animals. In developing countries, mortality rates are as high as 28 per 100,000 ; in North America the rate is less than 0.1 per 100,000 . Immunization programs clearly decreased neonatal tetanus deaths, and some recent evidence suggests progress in prevention throughout the World. Tetanus remains a great public health problem in developing countries such as Nigeria and it is associated with high mortality, but the understanding of the epidemiological aspects of this disease forms the basis of preventive strategies in any community. This paper reviewed the history, epidemiology and clinical diagnosis of tetanus and emphasized the current opinions in the management of tetanus outlining the various suggested treatment plans from experts in developing countries which form the basis for World Health Organization recommendations.

\section{INTRODUCTION}

Tetanus, an infective intoxication of the nervous system by clostridium tetani, is an ancient disease - as old as humans. It is currently responsible for 1.2 million deaths in the developing world. Despite the widespread availability of a safe and effective vaccine against this disease, it remains a major health problem in developing countries, and also occurs in countries with good standard of medical practice. It is a disease associated with a high mortality rate and a major cause of death among neonates in developing African countries. It typically presents with trismus, difficulty in swallowing and more ominously difficulty in breathing or opisthotonus. The incidence of tetanus has been on gradual and continuous decline in developed countries due to organized mass tetanus immunization campaigns. The widespread use of tetanus toxoids (formulated as diphteria, whole-cell pertusis vaccine and tetanus toxoid-DPT) for infants and children, the use of both tetanus toxoids and tetanus immune globulin (TIG) for post-exposure prophylaxis in wound treatment and improved care 
and management have contributed in no small way to reduction in developed countries.

\section{HISTORICAL PERSPECTIVE}

The word tetanus comes from the Greek tetanos, which is derived from the term teinein, meaning to stretch. Tetanus appears in military medical documents throughout the ages. In the Corpus Hippocraticum, Hippocrates $(460-170 \mathrm{BC})$ wrote that patients 'die within 4 days or if they pass these they recover'. Cappadocium Aretans, the second century physician, described tetanus as 'an awful disease, humble to watch' and he concluded that' the patient should die soon in order to end pain and wretched evil together with life'. Slapping infected dung on the umbilical cords of newborns (as part of ritualistic ceremonies) caused rampant tetanus neonatorum or trismus nascentium in the West Indies and in Africa. Osler's textbook describes the "eight days sickness" caused by umbilical sepsis, which killed 84 of 125 children within a fortnight of birth in St Kilda, Scotland. During World War I, tetanus occurred in 1.47 per 1000 British wounded and in 12.5 per 1000 persons involved in the Peninsular campaign. Nicolaier discovered the anaerobic bacillus Clostridium tetani in 1885 ending an era of ignorance of the causation. In 1889, Koch's pupil, Kitasato, obtained the bacillus of tetanus in pure culture and associated the disease to animals. The nineteenth and twentieth centuries however witnessed rapid and significant advances in the understanding of the pathophysiology of tetanus and this brought about the introduction of tetanus toxoid vaccination by Behring and Knorr in 1886.

\section{EPIDEMIOLOGY}

Today, tetanus remains a great public health problem in developing African countries such as Nigeria. It is an uncommon disease in most developed countries, where when it is reported it is associated with a high case fatality. Epidemiological studies have shown that tetanus affects all age groups but the predilection for the younger or older groups depends on whether the reports are from the developed or developing world. There are conflicting reports as to the seasonal variation in the prevalence of tetanus. While some workers report that it is more prevalent in the hot, dry season, others report that it is more common in the wet or humid seasons. The incidence of tetanus in Nigeria is higher in the hot dry season than in the wet but a few studies reported occurrence of more cases in the wet season. It is believed that the reduced incidence during the rainy season is due to reduced outdoor activity, increased wearing of shoes and the washing away of animal waste that sometimes litters the streets. On the other hand, the cases observed during the wet seasons were attributed to occupations of affected patients like farmers, manual laborers and traders as these groups of people are more prone to sustaining injuries during the wet season.

Several portals of entry have been documented, but in about $20 \%$ of cases, sources of infection may be unknown. Puncture wounds, circumcision, infected burns, postabortal sepsis, injection sites, guineaworn sores, road traffic accident wounds, otitis media, surgical procedures through contaminated gloves or poorly sterilized catgut sutures are some of the portals that have been described.

Tetanus continues to be a severe disease primarily of neonates 
and older adults who are unvaccinated or inadequately vaccinated In a study reported among a Danish population between 1978 and $1982^{5}$, only $10 \%$ had history of previous complete vaccination and a similar report from the United State between 1989 and 1990 showed that $11 \%$ had immunization prior to disease onset, and $31 \%$ lacked a history of tetanus vaccination. In the developed countries, earlier studies at the beginning of the twentieth century revealed a higher prevalence of tetanus in the lower age groups but more recently reports are showing a change with more cases being seen in the late adulthood and among the elderly people. This is attributed to the declining immune status of the elderly population. In the developing countries however, most cases are still in the younger age group although there are cases at the extremes of life, usually associated with higher mortality rates. In a study conducted in Benin City Nigeria, tetanus was more prevalent among the younger age group - a group commonly afflicted because of their susceptibility to injuries. However in the developed countries where the tetanus vaccination programs have been very effective, most patients are $>/=60$ year. This is related to declining immunity against tetanus as age increases. Data obtained from various national population-based serologic surveys indicate that the prevalence of immunity to tetanus is lower in older age groups, for example from $>70$ $80 \%$ among persons aged $6-39$ years declining to $28 \%$ among persons aged $>/=70$ years Studies have also shown that older adults lack protective levels of anti-tetanus antibodies and that elderly persons who reside in rural areas are more likely to be unimmunized than elderly persons in urban areas.
The sex difference varies from one environment to the other, probably due to differences in cultural practice. Some earlier studies in Nigeria have reported a preponderance of males and this was thought to be due to susceptibility of men to injuries by virtue of their occupation, as more men are engaged in farming than women. However this occupational practice is not true for some areas of Nigeria, e.g. Niger Delta where more women engage in farming than men, hence more women are exposed to farming injuries. This probably explained the higher female to male ratio in the study reported by Ogunrin et al as the Niger Delta area falls into the catchments area of the centre where this study was done.

Majority of tetanus cases reported in Nigeria occurred after an acute injury mainly in the feet and leg and the injuries were frequently sustained through stepping on wood and cuts from cutlass. This is similar to reports from the United States, where the most frequent wounds in tetanus patients were sustained by stepping on a nail Chen et al in China and Simmonsen et al in Denmark have also reported the same trend. These findings emphasized the place of wearing of shoes/boots or protective clothing by those who are in vulnerable positions by reason of their occupation as a mean of reducing the frequency of wounds sustained in such occupations.

The mortality of tetanus reported in the literature varies depending on the center where study was done. The reports on mortality from tetanus in developing countries vary. In Nigeria, the mortality rate varies from $38 \%$ to $60 \%$, depending on the infrastructure facilities and personnel at the reporting center $\mathrm{A}$ mortality rate of $26 \%$ was reported from a hospital-based study in Benin 
City which is comparable to the rate of $28 \%$ reported by Polish workers in 1972 but much higher than reports from western countries. Prognosis of tetanus varies according to duration of incubation, the speed with which signs become generalized, the severity of clinical presentation and access to the suitable treatment regimen. The average case-fatality ratio is approximately $50 \%$. Mortality is caused by infection, respiratory, cardiovascular or metabolic complications.

\section{CLINICAL PRESENTATION}

Tetanus is classically divided into four clinical types: generalized, localized, cephalic, and neonatal. These are valuable diagnostic and prognostic distinctions, but they reflect host factors and the site of inoculation rather than differences in toxin action. Terms describing the initial stages of tetanus include incubation period (time from inoculation to the first symptom which is usually trismus) and period of onset (time from the first symptom to the first generalized spasm). The shorter these periods are the worse the prognosis. Various rating scales are available (see scale below - table 1). Certain portals of entry (e.g., compound fractures) are associated with poorer prognoses. Tetanus may be particularly severe in narcotic addicts, for unknown reasons.

Generalized tetanus is the most commonly recognized form; it often begins with trismus (masseter rigidity, known as "lockjaw") and a risus sardonicus (increased tone in the orbicularis oris). Dysphagia and abdominal rigidity may also be present. The generalized spasm resembles decorticate posturing and consists of opisthotonic posturing with flexion of the arms and extension of the legs. The patient does not lose consciousness and experiences severe pain during each spasm. The spasms often are triggered by sensory stimuli. During the spasm, the upper airway can be obstructed, or the diaphragm may participate in the general muscular contraction. Either of these compromises respiration and even the first of such spasm may be fatal. In the modern era of intensive care, however, the respiratory problems are easily managed, and autonomic dysfunction, usually occurring after several days of symptoms, has emerged as the leading cause of death. The illness can progress for about 2 weeks, reflecting the time required to complete the transport of toxin that is already intraaxonal when antitoxin treatment is given. The severity of illness may be decreased by partial immunity. Recovery takes an additional month and is complete unless complications supervene. Lower motor neuron dysfunction may not be apparent until spasms remit, and recovery from this deficit in neuromuscular transmission may take additional weeks. Recurrent tetanus may occur if the patient does not receive active immunization, because the amount of toxin produced is inadequate to induce immunity.

Localized tetanus involves rigidity of the muscles associated with the site of spore inoculation. This may be mild and persistent and often resolves spontaneously. Lower motor neuron dysfunction (weakness and diminished muscle tone) is often present in the most involved muscle. This chronic form of the disease probably reflects partial immunity to tetanospasmin. However, localized tetanus is more commonly a prodrome of generalized tetanus, which occurs when enough toxin gains access to the central nervous system.

Cephalic tetanus is a special form of localized disease affecting the cranial nerve musculature. Although 
earlier reports linked cephalic tetanus to a poor prognosis, more recent studies have revealed many milder cases. A lower motor neuron lesion, frequently producing facial nerve weakness, is often apparent. Extraocular muscle involvement is occasionally noted.

\section{DIAGNOSIS}

Tetanus is diagnosed by clinical observation and has a limited differential diagnosis. Laboratory testing cannot confirm or exclude the condition, and it is primarily useful for excluding intoxications that can mimic tetanus. Electromyographic studies are occasionally useful in questionable cases. Such testing becomes more important when no portal of entry is apparent. Antitetanus antibodies are undetectable in most tetanus patients, but many reports document the disease in patients with antibody levels above the commonly cited "protective" concentration of $0.01 \mathrm{IU} / \mathrm{L}$. Rare patients apparently develop antibodies that are not protective.

Attempts to culture C. tetani from wounds are not useful in diagnosis, since (1) even carefully performed anaerobic cultures are frequently negative; (2) a positive culture does not indicate whether the organism contains the toxin-producing plasmid; and (3) a positive culture may be present without disease in patients with adequate immunity.

Strychnine poisoning, in which glycine is antagonized, is the only condition that truly mimics tetanus; toxicologic studies of serum and urine should be performed when tetanus is suspected, and tetanus should be considered even if strychnine poisoning appears likely. Because the initial treatments of tetanus and strychnine intoxication are similar, therapy is instituted before the assay results are available. Dystonic reactions to neuroleptic drugs or other central dopamine antagonists may be confused with the neck stiffness of tetanus, but the posture of patients with dystonic reactions almost always involves lateral head turning, which is rare in tetanus. Treatment with anticholinergic agents (benztropine or diphenhydramine) is rapidly effective against dystonic reactions. Dental infections can produce trismus, and should be sought, but they do not cause the other manifestations of tetanus.

Table 1: ABLETT CLASSIFICATION OF SEVERITY OF TETANUS

\begin{tabular}{|c|c|}
\hline Grade & Clinical features \\
\hline I & $\begin{array}{l}\text { Mild: mild to moderate trismus; general spasticity; no respiratory } \\
\text { embarrassment; no spasms; little or no dysphagia. }\end{array}$ \\
\hline II & $\begin{array}{l}\text { Moderate: moderate trismus; well-marked rigidity; mild to moderate but short } \\
\text { spasms; moderate respiratory embarrassment with an increased respiratory } \\
\text { rate greater than } 30 ; \text { mild dysphagia. }\end{array}$ \\
\hline III & $\begin{array}{l}\text { Severe: severe trismus; generalized spasticity; reflex prolonged spasms; } \\
\text { increased respiratory rate greater than } 40 \text {; apnoeic spells; severe } \\
\text { dysphagia; tachycardia greater than } 120 .\end{array}$ \\
\hline IV & $\begin{array}{l}\text { Very severe: grade III and violent autonomic disturbances involving the } \\
\text { cardiovascular system. Severe hypertension and tachycardia alternating } \\
\text { with relative hypotension and bradycardia, either of which may be } \\
\text { persistent. }\end{array}$ \\
\hline
\end{tabular}

A prognostic score has been proposed by a team from Senegal (Dakar, 1975), which can be established after the third day following the first clinical signs. 
The prognosis will be bleaker as the clinical score increases.

\begin{tabular}{|c|c|c|c|}
\hline Number & Prognostic factor & 1 point & 0 point \\
\hline 1 & $\begin{array}{l}\text { Duration of } \\
\text { incubation }\end{array}$ & $<7$ days & $\begin{array}{l}\geq 7 \text { days or } \\
\text { unknown }\end{array}$ \\
\hline 2 & Duration of patency & $<2$ days & $\geq 2$ days \\
\hline 3 & Entry point & $\begin{array}{l}\text { Ombilicus, uterus, burns, } \\
\text { complex open fractures, } \\
\text { surgical procedure, } \\
\text { intramuscular injection }\end{array}$ & $\begin{array}{l}\text { Other or unknow } \\
\text { point of entry }\end{array}$ \\
\hline 4 & Paroxysms & Yes & No \\
\hline 5 & Rectal temperature & $>38.4^{\circ} \mathrm{C}$ & $\leq 38.4^{\circ} \mathrm{C}$ \\
\hline 6 & $\begin{array}{l}\text { Pulse } \\
\begin{array}{l}-\quad \text { in adults } \\
-\quad \text { in newborns }\end{array}\end{array}$ & $\begin{array}{l}>120 \mathrm{bpm} \\
>150 \mathrm{bpm}\end{array}$ & $\begin{array}{l}\leq 120 \mathrm{bpm} \\
\leq 150 \mathrm{bpm}\end{array}$ \\
\hline
\end{tabular}

The disease progresses over 3 weeks on average. Disease may recur in survivors who have not been vaccinated, as the infection is not immunogenic.

\section{MANAGEMENT}

The patient with tetanus requires simultaneous attention to several concerns. Attention to the airway and to ventilation is paramount at the time of presentation, but the other aspects of care, especially passive immunization, must be pursued as soon as the respiratory system is secure. The goals of therapy are to eliminate the source of toxin, neutralize unbound toxin and prevent or abort (as the case may be) muscle spasms, monitoring the patient's condition and providing support especially respiratory support - until recovery. Table 1 presents a suggested management protocol.

\section{a. Eliminating the source of toxin:} Antibiotic Therapy

Although of unproven value, antibiotic therapy is administered to eradicate vegetative cells - the source of toxin. The in vitro susceptibilities of C. tetani include metronidazole, penicillins, cephalosporins, imipenem, macrolides, and tetracycline. The use of penicillin (10 to 12 million units intravenously, given daily for 10 days) has been recommended, but metronidazole (500 mg every $6 \mathrm{~h}$ or 1 $\mathrm{g}$ every $12 \mathrm{~h}$ ) is preferred by some experts on the basis of this drug's excellent antimicrobial activity, a survival rate higher than that obtained with penicillin in one nonrandomized trial, and the absence of the GABA antagonistic activity seen with penicillin. A study comparing oral metronidazole with intramuscular penicillin showed a better survival rate, shorter hospitalization, and less progression of disease in the metronidazole group. Another prospective, open, non-randomised clinical trial was carried out to compare the efficacy of procaine penicillin with metronidazole in the treatment of moderate tetanus among 173 patients. Patients in the metronidazole group had a significantly lower mortality rate, a shorter stay in hospital, and an improved response to treatment. These results showed that metronidazole is more efficacious than penicillin in this respect. Clindamycin and erythromycin are also alternatives for the treatment of penicillin-allergic patients. Additional specific antimicrobial therapy should be given for active infection with other organisms. In patients with a deep wound, thorough debridement and toilet, and antibiotic therapy are critical to remove the source of toxin 
production. If the wound itself requires surgical attention, this may be performed after spasms are controlled. However, the course of tetanus is not affected by wound débridement though a contrary observation was noted in a study that showed a 1.5 odds ratio in favour of patients who had wound debridement. Topical antibiotic application to the umbilical stump appears to reduce the risk of neonatal tetanus.

\section{b. Neutralizing unbound toxin}

Antitoxin is used to neutralize circulating toxin and unbound toxin in the wound, antitoxin effectively lowers mortality, with an odds ratio of 4.56; toxin already bound to neural tissue is unaffected. Passive immunization with human tetanus immune globulin (HTIG) shortens the course of tetanus and may lessen its severity. Human tetanus immune globulin (TIG) is the preparation of choice and should be given promptly. The dose is 3000 to 6000 units intramuscularly, usually in divided doses because the volume is large. The optimal dose is not known, however, and results from one study indicated that a 500-unit dose was as effective as higher doses. Pooled intravenous immunoglobulin may be an alternative to TIG, but the specific antitoxin concentration in this formulation is not standardized. There is no apparent advantage to intrathecal HTIG administration. Intrathecal (HTIG) has also been shown to be ineffective in neonatal tetanus. Pooled intravenous immune globulin has been proposed as an alternative to HTIG. It may be best to administer antitoxin before manipulating the wound; the value of injecting a dose proximal to the wound or infiltrating the wound is unclear. Additional doses are unnecessary because the half-life of antitoxin is long. Antibody does not penetrate the blood-brain barrier. Intrathecal administration should be considered experimental. Equine tetanus antitoxin (TAT) is not available in the United States but is used elsewhere especially in poor resource countries. It is cheaper than human antitoxin, but its half-life is shorter and its administration commonly elicits hypersensitivity and serum sickness. Active immunization must also be initiated after antitoxin.

\section{c. Control of Muscle spasms}

Many agents, alone and in combination, have been used to treat the muscle spasms of tetanus, which are painful and can threaten ventilation by causing laryngospasm or sustained contraction of ventilatory muscles. The ideal therapeutic regimen would abolish spasmodic activity without causing oversedation and hypoventilation. Benzodiazepines have emerged as the mainstay of symptomatic therapy for tetanus. These drugs are GABA agonists and thereby indirectly antagonize the effects of the toxin. They do not restore glycinergic inhibition. Diazepam, a benzodiazepine and GABA agonist, is in wide use. The dose is titrated, and large doses (250 $\mathrm{mg} / \mathrm{d}$ ) may be required. Lorazepam, with a longer duration of action, and midazolam, with a short half-life, are other options. Barbiturates and chlorpromazine are considered second-line agents and seem equally effective. Therapeutic paralysis with a non-depolarizing neuromuscular blocking agent and mechanical ventilation may be required for the treatment of spasms unresponsive to medication or spasms that threaten ventilation. Tetanus patients have unusually high tolerance for the sedating effect of these agents and commonly remain alert at doses normally expected to produce anaesthesia. However, prolonged paralysis after the discontinuation of therapy with such agents has been 
described, and both the need for continued paralysis and the occurrence of complications should be assessed daily. After the symptoms of tetanus subside, these agents must be tapered over at least 2 weeks to prevent withdrawal effects. Alternative agents include propofol, which is expensive, and dantrolene and baclofen, which are being investigated in the hope of shortening the period of therapeutic paralysis. Propofol infusion is also effective but very expensive, and the amount necessary to control symptoms may exceed the patient's tolerance of the lipid vehicle. Neuroleptic agents and barbiturates, previously used for tetanus, are inferior for this indication and should not be used. Magnesium infusion is rapidly emerging as a useful therapeutic technique in generalized tetanus.

The intravenous formulations of both diazepam and lorazepam contain propylene glycol; at the doses required to control generalized tetanus, this vehicle may produce lactic acidosis. Nasogastric delivery of these agents is possible but often discouraged because of risk of laryngospasm and some tetanus patients develop gastrointestinal motility disorders and do not absorb drugs well. Intravenous midazolam (5 to $15 \mathrm{mg} / \mathrm{hour}$ or more) is effective and does not contain propylene glycol, but it must be given as a continuous infusion because of its brief half-life. Rarely, tetanus cannot be adequately controlled with benzodiazepines alone; neuromuscular junction blockade is then indicated, with the caveat that sedation is still required for psychological reasons. All of the available drugs have side effects, including the potential for prolonged effects after the drug is discontinued. Vecuronium (by continuous infusion) or pancuronium (by intermittent injection) is an adequate choice. These agents should be stopped at least once daily to assess the patient's progress and to observe for possible complications.

Electroencephalographic monitoring is a useful adjunct for this purpose.

\section{d. Monitoring the patient's condition and providing support - especially respiratory support - until recovery.}

Tetanic spasms sometimes demand that the airway be secured before other lines of therapy are possible. Intubation or tracheostomy, with or without mechanical ventilation, may be required for hypoventilation due to oversedation or laryngospasm or for the avoidance of aspiration by patients with trismus, disordered swallowing, or dysphagia. The need for these procedures should be anticipated, and they should be undertaken electively and early. An orotracheal tube can be passed under sedation and neuromuscular junction blockade; a feeding tube should be placed at the same time. Please note that because the endotracheal tube can stimulate spasms, an early tracheostomy may be beneficial.

\section{e. Autonomic Dysfunction}

The optimal therapy for sympathetic overactivity has not been defined. Autonomic dysfunction generally reflects excessive catecholamine release and may respond to combined $\alpha-$ and $\beta$ adrenergic blockade with intravenous labetalol. Agents that have been considered include labetalol (an $\alpha$ - and $\beta$-adrenergic blocking agent that is recommended by some experts but that reportedly has caused sudden death), esmolol administered by continuous infusion (a beta blocker whose short half-life may be advantageous in the event of severe hypertension from unopposed $\alpha$ adrenergic activity), clonidine (a 
central-acting antiadrenergic drug), and morphine sulphate. Parenteral magnesium sulfate and continuous spinal or epidural anesthesia have been used but may be more difficult to administer and monitor. The relative efficacy of these modalities has yet to be determined. Hypotension or bradycardia may require volume expansion, use of vasopressors or chronotropic agents, or pacemaker insertion.

Other approaches to hypertension include morphine infusion, magnesium sulfate infusion, and epidural blockade of the renal nerves. Hypotension is less common; if present, it may require norepinephrine infusion. Myocardial dysfunction is also common and may represent a further reflection of catecholamine excess.

Nutritional support should be started as soon as the patient is stable. The volume of enteral feeding needed to meet the exceptionally high caloric and protein requirements of these patients may exceed the capacity of the gastrointestinal system.

\section{Table II: SUGGESTED MANAGEMENT PROTOCOL FOR GENERALIZED TETANUS (modified by author to suit local management strategies)}

\section{Diagnosis and stabilization: first hour after presentation}

A. Assess airway and ventilation. If necessary, perform endotracheal intubation using benzodiazepine sedation and neuromuscular blockade (e.g., vecuronium $0.1 \mathrm{mg} / \mathrm{kg}$ ). This is not necessary in most cases.

B. Obtain samples for antitoxin level, strychnine and dopamine antagonist assays, electrolytes, blood urea nitrogen, creatinine, creatine kinase, and urinary myoglobin determination.

C. Determine the portal of entry, incubation period, period of onset, and immunization history.

D. Administer benztropine (1-2 mg, intravenously) or diphenhydramine (50 mg, intravenously) to rule out a dystonic reaction to a dopamine blocking agent (if not sure of diagnosis). In most cases however the diagnosis is obvious.

E. Administer a benzodiazepine intravenously (diazepam in 5-mg increments, or lorazepam in 2-mg increments) to control spasm and decrease rigidity. Initially, employ a dose that is adequate to produce sedation and minimize reflex spasms. If this dose compromises the airway or ventilation, intubate using a short-acting neuromuscular blocking agent. Transfer the patient to a quiet, darkened area of the intensive care unit.

\section{Early management phase: first 24 hours}

A. Administer human tetanus immunoglobulin (HTIG), $500 \mathrm{IU}$, intramuscularly; as an alternative, consider intravenous pooled immune globulin (see text). In some African countries including Nigeria, the equine anti-tetanus serum is used because it is cheap and readily available.

B. At a different site, administer adsorbed tetanus toxoid such as tetanus-diphtheria vaccine $(0.5 \mathrm{ml})$ or diphtheria-pertussis-tetanus vaccine $(0.5 \mathrm{ml})$, as appropriate for age, intramuscularly. Adsorbed tetanus toxoid without diphtheria toxoid is available for patients with a history of reaction to diphtheria toxoid; otherwise, the correct combination for the patient's age should be employed. This is controversial as some experts believe active immunization should be commenced when the patient is more stable or prior to discharge from the hospital. This is to prevent the possible neutralizing effect of TIG on the tetanus toxoid.

C. Begin metronidazole $500 \mathrm{mg}$, intravenously, every $6 \mathrm{~h}$ for $7-10$ days. Alternatively intravenous penicillin may be given (ensure patient is not allergic to penicillin).

D. Perform a tracheostomy after placement of an endotracheal tube and under neuromuscular blockade if spasms produce any degree of airway compromise.

E. Débride wounds as indicated.

F. Place a soft, small-bore nasal feeding tube or a central venous hyperalimentation catheter, and 
begin feeding. Some physicians prefer to place patients on nothing per oral until spasms are controlled to avoid the risk of laryngospasm. Patients receiving total parental nutrition should be given parenteral $\mathrm{H}_{2}$ blockade or other gastric protection.

$\mathrm{G}$. Administer benzodiazepines as required to control spasms and produce sedation. If adequate control is not achieved, institute long-term neuromuscular blockade (e.g., vecuronium 6 to $8 \mathrm{mg} / \mathrm{h}$ ); continue benzodiazepines for sedation with intermittent electroencephalographic monitoring to ensure somnolence. Neuromuscular junction blockade should be discontinued daily to assess the patient's physical examination and to decrease the possibility of excessive accumulation of the blocking agent.

\section{Intermediate management phase: next 2-3 wk}

A. Treat sympathetic hyperactivity with labetalol $(0.25-1.0 \mathrm{mg} / \mathrm{min}$ as needed for blood pressure control) or morphine (0.5-1.0 mg/kg/h by continuous infusion; see text for other recommendations). Consider epidural blockade with a local anesthetic. Avoid diuretics for blood pressure control, because volume depletion worsens autonomic instability.

$B$. If hypotension is present, initiate saline resuscitation. Place a pulmonary artery catheter and an arterial line and administer fluids, dopamine, or norepinephrine as indicated.

C. Sustained bradycardia usually requires a pacemaker. Atropine or isoproterenol may be useful during pacemaker placement.

D. Begin prophylactic heparin

E. Use a flotation bed, if possible, to prevent skin breakdown and peroneal nerve palsies. Otherwise, ensure frequent turning and employ antirotation boots.

F. Maintain benzodiazepines until neuromuscular blockade, if employed, has been terminated and the severity of spasms has diminished substantially. Then taper the benzodiazepine dose over 14-21 days.

G. Begin rehabilitation planning.

IV. Convalescent stage: 2-6 weeks

A. After spasms are no longer present, begin physical therapy. Many patients require supportive psychotherapy.

B. Before discharge, administer another dose of tetanus-diphtheria vaccine or diphtheria-pertussistetanus vaccine.

C. Schedule a third dose of toxoid to be given 4 weeks after the second.

Adapted from Bleck TP, Brauner JS. Tetanus. In: Scheld WM, Whitley RJ, Durack DT, eds. Infections of the Central Nervous System. 2nd ed. Philadelphia: Lippincott-Raven; 1997:629-653.

\section{Prognosis}

The severity of spasms, duration of hospital stay, incubation period, onset time, immunization status and socio-economic background and the type of initial care are documented factors that affect mortality. The impact of pre-hospital care on outcome of tetanus infection needs to be further investigated. Prognosis has become progressively more favourable in developed countries. The mortality rate of mild and moderate tetanus in Western countries is presently about $6 \%$; for severe tetanus, it may reach as high as $60 \%$, even in expert centers.
Among adults, age has very little effect on mortality, with octogenarians and nonagenarians faring as well as middle-aged patients. Tetanus survivors often have serious psychological problems related to the disease and its treatment that persist after recovery and may require psychotherapy.

The course of tetanus extends over 4 to 6 weeks, and patients may require ventilatory support for 3 weeks during this period. Increased tone and minor spasms can last for months, but recovery is usually complete. In a series of Italian patients, the mean length of stay was 40.3 days. In an 
American series (CDC), previous vaccination status was directly related to severity of disease, with the casefatality ratio ranging from $6 \%$ for patients who had received one to two doses to $15 \%$ for patients who were unvaccinated. No deaths occurred among the 16 patients who previously had received three or more doses.

\section{PROPHYLAXIS}

\section{Active Immunization}

Tetanus is preventable in almost all patients, leading to its description as the "inexcusable disease." A series of three monthly intramuscular injections of alumadsorbed tetanus toxoid provides almost complete immunity for at least 5 years. Active protection should be maintained by administrating booster doses every 10 years. Patients recovering from tetanus should be actively immunized (see below) because immunity is not induced by the small amount of toxin that produces disease.

Although a full series of maternal immunizations is ideal, even one or two doses of tetanus toxoid confer substantial protection against neonatal tetanus In countries with incomplete immunization programs for children, all pregnant women should receive 2 doses of tetanus toxoid during the first pregnancy, with an interval of at least 1 month, and with the second dose at least 2 weeks before child birth. Application of topical antimicrobial agents to the umbilical cord stump markedly decreases the incidence of neonatal tetanus when maternal immunization is insufficient.

Some patients with humoral immune deficiencies may not respond adequately to toxoid injection; such patients should receive passive immunization for tetanus-prone injuries regardless of the period since the last booster injection.

\section{Prophylaxis in wound management}

Although any wound may be inoculated with tetanus spores, some types of injury are more frequently associated with tetanus (tetanus prone). These include wounds that are contaminated with dirt, saliva, or feces; puncture wounds, including unsterile injections; missile injuries; burns; frostbite; avulsions; and crush injuries. Patients with these wounds who have not received adequate active immunization in the past 5 years, or in whom immunodeficiency is suspected, should receive passive immunization with Human TIG (250 to 500 IU, intramuscularly, regardless to the patient's age) in addition to active immunization. If globulin is not available, animal antitoxin could be used at the dose of 1500 to $5000 \mathrm{IU}$. In that case, it is essential to avoid anaphylaxis by first injecting $0.02 \mathrm{ml}$ of a $1 / 100$ dilution in physiological saline solution intradermally, with a syringe containing adrenaline on hand. It is recommended to pretest with 1/1000 solution if there has been prior animal serum exposure, with a similar saline solution injection as a negative control. If after 15-20 min there is a wheal with surrounding erythema, at least $3 \mathrm{~mm}$ larger than the control injection site, it is necessary to desensitize the individual.

Neonatal tetanus: Measures aimed at preventing neonatal tetanus include maternal vaccination, even during pregnancy; efforts to increase the proportion of births that take place in the hospital; and the provision of training for nonmedical birth attendants.

Early human tetanus
immunoglobulin administrations are associated with decreased mortality. Once the toxin is bound and internalized it will have no effect. When 3000 IU are administered intramuscularly, 
concentrations reach in 24-48 $\mathrm{hr}$ and approximately $0.1 \mathrm{IU} / \mathrm{mL}$. Adequate concentrations of passive antitoxin that protect a human against tetanus are maintained for 10-15 days. Patients with tetanus also should actively be immunized through administration of adsorbed tetanus toxoid given intramuscularly at a site contralateral to the HTIG dosing. In a Korean study, vaccination with lower dose of DT $(0.25 \mathrm{~mL})$ induced an excellent immune response. In a monocentric, double-blind, randomized trial examining the safety and pharmacokinetic profile of a new, pasteurized, human tetanus immunoglobulin (P-HTIG) and $\mathrm{Td}$ vaccine in combination in humans, the evolution of the tetanus antibody titers in the group that received P-HTIG alone and in the group that received $\mathrm{P}$ HTIG plus Td vaccine was similar until D3 to D7. In the P-HTIG plus placebo group, tetanus antibody titers rose to a maximum of $0.31362 .49 \mathrm{IU} / \mathrm{ml}$ after 4.4 days; in the P-HTIG plus $\mathrm{Td}$ vaccine group, a maximum concentration of $15.262 .42 \mathrm{IU} / \mathrm{ml}$ was reached 19 days postinjection. In both groups, $100 \%$ of the patients had seroprotective levels of tetanus antibodies $(>0.01 \mathrm{IU} / \mathrm{ml}) 2$ days following treatment. An anamnestic response to $\mathrm{Td}$ vaccine appeared 7 days postimmunization. In conclusion, P-HTIG has a good safety and pharmacokinetic profile. Our results confirm that immunoglobulin should be associated with vaccine in the treatment of tetanus-prone wounds. Likewise, modelization of antibody titers clearly showed similar profiles in the two groups until the seventh day. These results demonstrate the absence of an early antitoxin response to the tetanus booster, similar to previous observations. The authors estimate that the formation of antibodies in response to vaccination occurs at about day 6. (An exact prediction cannot be made because of insufficient data between days 3 and 7.) Thus, treatment of tetanus-prone wounds with immunoglobulin is crucial, especially in light of the fact that the incubation period of tetanus can be as short as 3 to 4 days.

Although data are conflicting, concurrent malarial infection may decrease the immune response to TT; however, malarial chemoprophylaxis may enhance the immune response. Malnutrition does not appear to affect immunogenicity; nevertheless, one study suggests that vitamin $A$ deficiency is associated with an impaired immune response. Although it has been postulated that placental transfer of tetanus antibody is impaired in African women, a survey of the published literature suggests that this is not the case. Freezing TT has been shown to decrease its potency, but its impact on immunogenicity needs more evaluation. Conversely, poor storage conditions may reduce effectiveness: In the UK, a study found that safe storage of vaccine, and therefore guarantee of efficacy, is not occurring in the majority of A\&E departments. Unnoticed failure of refrigerators could be exposing patients to the risk of tetanus infection.

Table III: Management of tetanus in adult humans (African Study Group on Tetanus)

It may be best to administer antitoxin before manipulating the wound.

I) $\frac{\text { Human tetanus immune }}{\text { globulin (HTIG) }}$

3000-10 000 UI of HTIG by IM or IV route $(40-150 \mathrm{UI} / \mathrm{kg})$ according to most official recommendations

500 IU IM may be as effective as larger doses according to some sources.

Inject as soon as possible 
Large doses may be fractioned and injected at multiple sites

Site of injection proximal to the wound doesn't appear to be preferable.

No necessity for additional doses (because of prolonged half-life)

DO NOT USE IM HTIG BY THE IV ROUTE (anaphylaxis)

II) Antibiotics

Intravenous Metronidazole $500 \mathrm{mg}$ every 6 hours or $1 \mathrm{~g}$ every 12 hours for at least 10 days

$$
\text { Or }
$$

Penicillin 10-12 million units daily IV for 10 days

III) Benzodiazepine

Diazepam intravenously by $5 \mathrm{mg}$ increment to control spasm

Or

Barbiturates / Chlorpromazine

IV) Symptomatic treatment

Rehydration

Mechanical ventilation if needed

Nutritional support

Prophylactic heparin

V) Immunization

Active immunization is needed because immunity is not induced by the small amount of toxin produced by the disease.

\section{GUIDELINES FOR THE} MANAGEMENT OF TETANUS IN ADULT PATIENTS AT THE UBTH

Prognosis of tetanus varies according to duration of incubation, the speed with which signs become generalized, the severity of clinical presentation and access to the suitable treatment regimen. The average case-fatality ratio is approximately $50 \%$. Mortality is caused by infection, respiratory, cardiovascular or metabolic complications.

A. Administer serum/human anti-tetanus tetanus immune globulin (HTIG) - 10, 000 or 20,000 units of ATS or $3,000-10,000$ UI of HTIG by IM or IV route (40-150Ul/Kg of HTIG). 500IU may be as effective as larger doses. For HTIG - inject as soon as possible. Large doses may be fractionated and injected at multiple sites. Site of injection proximal to the wound does not appear to be preferable. No necessity for additional doses (due to long half-life).

DO NOT USE IM HTIG BY THE IV ROUTE (YOU WILL GET ANAPHYLAXIS)

B. Give antibiotics - intravenous metronidazole 500mg every 6-8 hours or $1 \mathrm{gm} 12$ hourly for at least 10 days OR Penicillin 1012 million units in divided doses daily for 10 days.

C. Give Benzodiazepine diazepam intravenously by $5 \mathrm{mg}$ increment to control spasm. May add barbiturates and/or metocarbamol. In exceptional cases add chlorpromazine.

D. Symptomatic treatment rehydration with dextrose/saline infusion, nutritional support, mechanical ventilation in ICU and prophylactic heparin.

E. Immunization - active immunization is needed because immunity is not induced by small amount of toxin produced by the organism. Give IM tetanus toxoid at discharge and repeat at 2 and 6 weeks after the initial dose.

From Neurology Unit, Department of Medicine, UBTH. Modified from the African Tetanus Study Group 2007

\section{CONCLUSION}

Tetanus remains a clinical diagnosis because confirmatory laboratory tests are not available for routine use. Isolation of organism from 
wounds is neither sensitive nor specific, because anaerobic cultures of tissues or aspirates usually are not positive and the organism might be grown from wounds in the absence of clinical signs and symptoms of disease ${ }^{21,22}$. The mortality associated with the infection is very high, especially in our environment where the lack of adequate facilities mitigates against comprehensive and optimal hospital in-patient care. Our salvation therefore lies in preventive strategies implementation. These include such measures as health education of the public on the need to protect themselves against sustaining wounds (especially by wearing protective shoes on the farm or the use of thick gloves for manual laborer), and the prompt treatment of every wound and the administration of tetanus toxoid and/ or tetanus immunoglobulin by a qualified health care giver. Other measures include the establishment of tetanus care units in tertiary health institution, the inclusion of tetanus management as part of continuing medical education for all health care providers by their respective professional bodies and the zealous and dedicated implementation of the National program on Immunization on the part of the government.

\section{REFERENCES}

1. Abrutyn, E. Tetanus. Section 143. Harrison's Principles of Internal Mediciene, 15th, ed. copyright. 2001 McGraw-Hill.

2. Adams EB, Lawrence DR, Smith JWG. Tetanus. Oxford, Blackwell Scientific Publication, 1969. Pg. 1

3. Adeuja AOG, Osuntokun BO. Tetanus in the adult Nigerian: a review of 503 patients. East
Afr. Med. J. 1971; 48: 653 691.

4. Adudu $\mathrm{O} \mathrm{P}$, Ogunrin $\mathrm{O} \mathrm{A}$, Adudu $O$ G. Morbidity and Mortality patterns among neurological patients in the intensive care unit of a tertiary health facility. Ann. Afr. Med. 2007; 6(4): 174-9.

5. Afonja $\mathrm{AO}$, Jaiyeola $\mathrm{BO}$, Tunwashe L. Tetanus in Lagos. A review of 503 adult Nigerian patients. J. Trop Med Hyg. 1973; 76:171 - 174.

6. Alfery DD, Rauscher LA. Tetanus: a review. Crit. Care Med. 1979; 7: 176-81.

7. Arogundade FA, Bello IS, Kuteyi EA, et al. Pattern of presentation and mortality in tetanus a 10 year retrospective review. Niger Postgrad Med J. 2004; 11(3): 198-202.

8. Bandele EO, Akinyanju MO, Bojuwoye BJ. An analysis of tetanus deaths in Lagos. J. Natl. Med. Assoc 1991; 83(1); 55 58.

9. Behring E. Knorr A. Tetanusantition fur dieAnwendung in der praxis. Dtsch.Med. Wschr. Monograph. 1896.

10.Bleck, TP. Clostridium tetani (Tetanus). Chapter 234. Mandell, Douglas and Bennett's Principles and Practice of Infectious Diseases, 5th edition. Copyright. 2000, 1995, 1990, 1985, 1979 by Churchill Livingstone. 
11. Bytchenko D. Geographical distribution of tetanus in the world 1951 - 1960. A review of the problem. Bull World Health Organization. 1966; 34: 71104.

12. Caban J. Patients with tetanus from South Eastern Poland in material from the Clinic of Infectious Diseases in Krakow in the years $1993-1992$. Przegl - Lek 1994: 51(11): 4657.

13. Campbell J. Tetanus: a major killer still at large. Afri Health. 2000; 22(4): 8-10.

14. CDC. Tetanus - United States, 1985 -1986. MMWR 1987; 36: 477-81.

15. CDC. Tetanus- United States, 1987 and 1988. MMWR 1990; 39-41.

16. Chen FC, Sheen-Chen SM, Chou FF. High incidence of Tetanus in the middle aged and elderly. Chang Keng-I-Hsueh 1990; 13(4):296-303.

17. Edmondson RS, Flowers $M$ W. Intensive care in tetanus management complication and mortality in 100 cases. Br. Med. J. 1979:1 1404-4.

18. Gergen PJ, McQuillan GM, Kiely $M$, et al: A population based serologic survey of immunity to tetanus in the United States N. Eng. J Med. 1995; 332:761-766.

19. Haberman E. Tetanus. In Vinker, P.J. Bruyn,G.W. Eds. Handbook of Clinical Neurology, Amsterdam, North Holland
Publishing Company, 1978, Vol.3 pgs 491 - 547.

20. Izurieta HS, Sutter RW, Strebel PM, et al: Tetanus Surveillance - United States, 1991-1994; 46 (SS- 2): 15-691.

21. Ogunrin AO, Unuigbe El, Azubuike CO. Characteristics of tetanus cases seen over a ten year period in a tertiary health facility in Benin City, Nigeria. Ann Biomed Sci. 2006; 5(1\&2): 44-51.

22. Ogunrin OA, Unuigbe El. Tetanus: an analysis of the prognosticating factors of cases seen in a tertiary hospital in a developing African country between 1990 and 2000. Trop doct. 2004 ; 34(4): $240-41$.

23. Ojini FI, Danesi MA. Mortality of tetanus at the Lagos University Teaching Hospital, Nigeria. Trop Doct. 2005; 35(3): 178-81.

24. Onwuekwe IO, Onyedum CC, Nwabueze AC. Experience with tetanus in a tertiary hospital in South East Nigeria. Niger J Med. 2008; 17(1): 50-52.

25. Osuntokun BO. Perspectives in Tetanus: the past two decades. A symposium on 'Recent Advances in Neuroscience' Dokita 1990; 19(1): 74-78.

26. PHLS. Communicable Diseases Surveillance Report. Br. Med. J 1986; 293:680 -2.

27. Prevots R, Sutter RW, Strebel $\mathrm{PM}$, et al: Tetanus - United State 1989 - 1990 CDC MMWR 1992; 41 (8); 1 - 9. 
28. Rosmini F, Wirz M, Gentili G, et al: Year of birth, sex and residence as 'determinants' of tetanus incidence and immunity in Italy. Eur. J Epidemiology 1987; 3 (4); $337-80$.

29. Sanya EO, Taiwo SS, Olarinoye $\mathrm{JK}$, et al. A 12-year review of adult tetanus managed at the University College Hospital, Ibadan, Nigeria. Trop Doct 2007; 37: 170-72.

30. Simmonsen O, Block AV, Heron I. Epidemiology of Tetanus in Denmark 1920-1982, Scand. J Infect. Dis 1987; 19 (4); 437 44.
31. Unuigbe EI, Ogunrin AO. Does Pre-hospital care affect the outcome of tetanus patients? an analysis of cases seen at a tertiary hospital in Nigeria. Nig Med Pract. 2003; 44(1): 19-21.

32. Vidal J, Taberner JL, Plausi P, et al: The prevalence of tetanus antibodies in the adult population of Catalonia. Med. Clin. Barc. 1992; 99(1): 6-8.

33. Walsh JA. Establishing Health Priorities in the Developing World. New York, United Nations Development Programme, 1998, pg 15. 